\title{
Génétique moléculaire des déficits en glucose-6-phosphate déshydrogénase (G6PD)
}

La G6PD est la première enzyme de la voie des pentoses phosphates, dont le rôle principal est de fournir le coenzyme NADPH nécessaire à nombre de réactions de biosynthèse et de détoxication. Son déficit atteint environ 400 millions de personnes et paraît donc le plus fréquent de tous les déficits enzymatiques humains. On en a décrit plus de 300 variants sur des .critères physico-chimiques. La G6PD normale existe sous deux formes principales : le variant $\mathrm{B}$, ubiquitaire, et le variant $A$, présent chez $25 \%$ des sujets de race noire, et qui se reconnaît par une mobilité électrophorétique plus grande vers l'anode. Les mutants déficients provoquent des symptômes de gravité variable, depuis les crises hémolytiques déclenchées par la prise de certains médicaments ou la consommation de fèves, jusqu'à une anémie hémolytique permanente. Les mutants les plus fréquents dérivent apparemment des formes principales : Aobservé chez environ $10 \%$ des Noirs, $\mathrm{B}$ - retrouvé surtout sur le pourtour méditerranéen, et dont l'homogénéité était mise en doute. Un troisième mutant, appelé Canton, est fréquent dans l'est de l'Asie. Les autres mutants sont beaucoup plus rares. Depuis quelques années, le clonage de l'ADNc de la G6PD a permis de déterminer la structure du gène et de préciser la séquence de la protéine code pour une protéine de 515 acides aminés [3]. Il est donc devenu possible de commencer à défricher cette véritable forêt de mutants.

On a d'abord confirmé le fait, connu depuis longtemps par l'analyse de la protéine, que la forme $\mathrm{A}+$ dérive de $\mathrm{B}+$ par le remplacement d'une asparagine par un acide aspartique [4]. On a ensuite analysé une série de mutants, fréquents ou rares, dont les caractéristiques sont résumées dans le Tableau I $[3,5,6]$. De ce tableau, qui pourrait connaître une extension rapide, se dégagent quelques élé- ments essentiels : (1) les deux grands groupes, $\mathrm{A}$ et $\mathrm{B}$, dérivent bien l'un de l'autre par une seule mutation, Asn $\rightarrow$ Asp, qui augmente la charge négative de la protéine-enzyme et rend compte de la migration électrophorétique plus anodique de la forme $A$. Chaque mutant présente une mutation unique dans la séquence des acides aminés de $\mathrm{B}+$, à l'exception de $\mathrm{A}-$ : celui-ci possède en effet à la fois la mutation caractéristique de $\mathrm{A}+$ et une mutation supplémentaire $\mathrm{Val} \rightarrow$ Met. Elle s'est donc, à l'origine, greffée sur une population A+;

\begin{tabular}{|c|c|c|}
\hline \multicolumn{3}{|c|}{$\begin{array}{l}\text { Tableau I } \\
\text { MUTATIONS OBSERVÉES DANS DIFFÉRENTS VARIANTS, } \\
\text { INDIQUANT LA POSITION ET LA NATURE } \\
\text { DES CHANGEMENTS D'ACIDES AMINES }\end{array}$} \\
\hline Variant & Position & Changement \\
\hline $\begin{array}{l}A+[3,4] \\
A-[5] \\
\text { Metaponto [3] } \\
\text { llesha [3] } \\
\text { Chatham [3] } \\
\text { Santiago de Cuba [3] } \\
\text { Seattle [6] } \\
\text { Mediterranéen [6, 3] } \\
\text { Sassari [6] } \\
\text { Cagliari [6] }\end{array}$ & $\begin{array}{r}126 \\
126 \\
68 \\
58 \\
156 \\
335 \\
447 \\
282 \\
188 \\
188 \\
188\end{array}$ & $\begin{array}{l}\text { Asn } \rightarrow \text { Asp } \\
\text { Asn } \rightarrow \text { Asp } \\
\text { Val } \rightarrow \text { Met } \\
\text { Asp } \rightarrow \text { Asn } \\
\text { Glu } \rightarrow \text { Lys } \\
\text { Ala } \rightarrow \text { Thr } \\
\text { Gly } \rightarrow \text { Arg } \\
\text { Asp } \rightarrow \text { His } \\
\text { Ser } \rightarrow \text { Phe } \\
\text { Ser } \rightarrow \text { Phe } \\
\text { Ser } \rightarrow \text { Phe }\end{array}$ \\
\hline
\end{tabular}

La G6PD B+ est prise comme référence. 
certains mutants, comme Seattle, ont été trouvés en plusieurs endroits (États-Unis, Grèce, Sardaigne). On ne sait encore s'il s'agit de néomutations indépendantes ou de migrations à partir d'un fondateur unique; (3) l'observation la plus originale [6] est que plusieurs mutants individualisés sur des critères physicochimiques, à vrai dire peut-être raffinés à l'extrême, s'avèrent relever de la même mutation. Cette démonstration a été faite en Sardaigne sur les mutants Sassari et Cagliari, qui se sont révélés identiques au variant méditerranéen ou B-, dont l'unicité apparaît désormais probable. A mesure que l'on élucidera la nature de la mutation dans un nombre croissant de déficits, le nombre de variants de la G6PD pourrait donc diminuer. La raison pour laquelle des mutations identiques donnent des protéines et une gravité clinique quelque peu différentes selon les familles reste à déterminer. Une dernière remarque: on n'a jusqu'ici jamais détecté de délétions du gène; il est probable que l'absence complète de cette activité enzymatique, présente à des niveaux divers dans tous les tissus, serait incompatible avec la survie.

J.C. D.

l. Persico MG, Viglietto G, Martini G, et al. Isolation of human glucose-6-phosphate dehydrogenase cDNA clones : primary structure of the protein and unusual 5' non-coding regions. Nucleic Acids Res 1986 ; 14 : 2511-22.

2. Takizawa TI, Huang IY, Ikuta T, Yoshida A. Human G6PD : primary structure and cDNA cloning. Proc Natl Acad Sci USA $1986 ; 83$ : 4157-61.

3. Vuillamy TJ, D'Urso M, Battistuzzi G, et al.Diverse point mutations in the human glucose-6-phosphate dehydrogenase gene cause enzyme deficiency and mild or severe hemolytic anemia. Proc Natl Acad Sci USA $1988 ; 85: 5171-5$.

4. Yoshida A. A single aminoacid substitution (Asn to Asp) between normal $(B+)$ and he common negro variant $(\mathrm{A}+)$ of human glucose-6-phosphate dehydrogenase. Proc Natl Acad Sci USA 1967; 57 : 835-41.

5. Hirono A, Beutler E. Molecular cloning and nucleotide sequence of CDNA for human G6PD variant A-. Proc Natl Acad Sci USA $1988 ; 85: 3951-4$.

6. De Vita G, Alcalay M, Sanpietro M, et al. Two point mutations are responsible for G6PD polymorphism in Sardinia. Am J Hum Genet 1989; 44 : 233-40.

$\mathrm{m} / \mathrm{s} n^{\circ} 6$ vol. 5 , juin 89

\section{BRÈVES}

Existe-t-il des récepteurs membranaires des nucléosomes? Les nucléosomes sont des structures nucléoprotéiques dans lesquelles un noyau d'histone (un octamère composé de quatre types moléculaires de ces protéines basiques) est entouré de deux tours du double brin d'ADN. Conformation normale de l'ADN nucléaire, le nucléosome n'est pas censé interagir jamais avec la membrane plasmique. Et pourtant, Laurent Jacob et des chercheurs parisiens de l'Institut Pasteur (groupe de Daniel Louvard), de l'institut Gustave-Roussy (groupe de Jean-Bernard Lepecq) et de l'hôpital Necker (groupe de Jean-François Bach) viennent - de façon détournée - de montrer que des récepteurs membranaires de ces structures existaient bien [1]. Les malades souffrant de lupus érythémateux aigu disséminé ont des anticorps anti-ADN et antinucléprotéines. Mis en présence d'histone et d'ADN, certains de ces anticorps reconnaissent, spécifiquement, semble-t-il, une protéine de $94 \mathrm{kDa}$ de la membrane cytoplasmique. En fait, les anticorps ont une affinité maximale pour les nucléosomes et se fixent sur des particules qui sont elles-mêmes liées au récepteur supposé... comportant la protéine de $94 \mathrm{kDa}$. La formation d'un semblable complexe ternaire récepteur-nucléosome-anticorps serait associée à l'internalisation de l'ensemble. Quel est le mécanisme du développement des anticorps antiADN et antinucléosomes dans le lupus ? Quel rôle jouent-ils ensuite dans la pathogénie des complications de cette maladie? Autant de questions largement ouvertes à discussion... comme celle du rôle normal de cette «bizarrerie» que sont des récepteurs externes pour une structure nucléaire!

[1. Jacob L, et al. Proc Natl Acad Sci USA 1989 (sous presse).]
Génétique moléculaire de la neuropathie amyloïde en Europe. La polyneuropathie amyloïde familiale de type I a été décrite tout d'abord dans le nord du Portugal ; les premières manifestations cliniques apparaissent habituellement entre 20 et 40 ans; l'atteinte neurologique évolue progressivement et aboutit à la mort environ en une dizaine d'années. La maladie se transmet selon le mode dominant autosomique. La substance amyloïde dérive d'un variant de la transthyrétine (ou préalbumine) caractérisé par une substitution de la méthionine à la place de la valine en position 30 ; la mutation ponctuelle correspondante peut être identifiée dans l'ADN génomique. Holt et al. [1] ont ainsi étudié 13 familles atteintes européennes. Dans dix familles (huit originaires de Chypre, une de Grèce et une de France), la mutation de la polyneuropathie amyloïde de type I a été mise en évidence. Dans sept des dix familles, le caractère génétique de la maladie n'avait pas été reconnu auparavant ; la mutation a été identifiée chez 16 membres asymptomatiques parmi les 43 examinés; deux d'entre eux ont plus de 50 ans, ce qui montre bien que la pénétrance de la maladie est variable et que certaines formes peuvent être lentement évolutives. La connaissance de ces formes pourrait réduire la justification du diagnostic prénatal, mais les risques de développer une maladie grave sont probablement supérieurs à $70 \%$ chez les sujets porteurs de la mutation. Dans les trois autres familles, la mutation n'a pas été détectée. On connaît cependant d'autres types de polyneuropathie amyloïde familiale, caractérisés par d'autres variants de la transthyrétine et d'autres mutations ponctuelles.

[1. Holt IJ, et al. Lancet 1989; 1 : 524-6.]
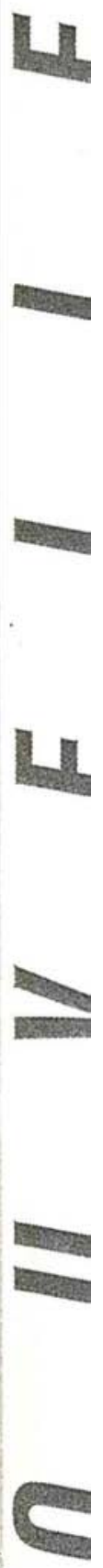